\title{
LES MASQUES
}

A la deuxième phase du mausolée, caractérisée par la construction du columbarium et de l'enclos funéraire, appartiennent quatre masques de pierre, dont trois furent découverts in situ (en m1, m2, m3 sur le plan fig. 2) le quatrième étant en remploi comme mascaron de dessus-de-porte dans une ferme voisine ${ }^{1}$. Mais par la qualité de la pierre et par la technique de sculpture, il appartient sans conteste à la série des trois premiers mis au jour pendant la fouille.

\section{MASQUE 1}

Fig. 43

Hauteur maximale : $77,5 \mathrm{~cm}$

Largeur de la base : $59 \mathrm{~cm}$

Hauteur du visage : $33 \mathrm{~cm}$

Epaisseur : $19 \mathrm{~cm}$

Ce masque d'homme aux traits accusés par l'âge, dont la coiffure est l'onkos traditionnel dans les rôles tragiques, est le mieux conservé ${ }^{2}$. La face arrière de la sculpture n'est pas travaillée et présente une courbure qui s'accentue à partir de la hauteur des yeux. L'onkos est fait de boucles symétriques en "anglaises", qui se superposent sur deux épaisseurs. Sur les côtés du visage, deux boucles descendent jusqu'au niveau de la barbe, tandis que sur le front, huit autres boucles, moitié moins longues, constituent l'essentiel de la coiffure. Le système des torsades est très régulier, chaque élément étant composé de quatre enroulements qui sont nettement séparés par un creux aménagé dans la pierre. Un bandeau, placé dans le tiers supérieur de la perruque et souligné par trois longs plis légèrement incisés, retient l'ensemble. Il retombe sur les côtés comme les pans d'un voile encadrant le visage. Un ruban plus mince apparaît également en haut du front. Le trait

1 La découverte est due à M. Y. Gautier, chargé de l'Inventaire pour la région des cantons de Pertuis et Cadenet. Le masque est fixé sur la façade de la ferme des Regrés, à une dizaine de kilomètres de Cucuron. Inventaire général, Pays d'Aigues, cantons de Cadenet et de Pertuis, p. 359, no 366 (cliché Heller, Roucaute). L'habitude de placer des têtes d'Hercule comme dessus de porte est fréquente en Provence, voir notamment les exemples du $\mathrm{xVII}^{\mathrm{e}} \mathrm{s}$. réunis pour Beaucaire : P. Neuman, Hercule à Beaucaire, Bulletin de la Sociélé d'Histoire et d'Archéologie de Beaucaire, 88, 1986, p. 13-15.

2 Cf. Informations archéologiques de Provence, Gallia, $30,1972,2$, p. 537, fig. 39-40. La dimension donnée en légende est erronée. majeur de la physionomie est donné par le pli hypertrophié des sourcils, très proéminents et qui dessinent deux larges fronces à la racine du nez. Ce dernier est assez fort, avec des narines importantes mais peu évidées. Les yeux, sous des paupières épaisses, ne comportent aucune trace de pupille, alors que le point lacrymal est profondément marqué en triangle, au coin interne de l'œil. Ce détail qui s'ajoute à la vigueur du bourrelet sourcilier, produit une curieuse impression de strabisme convergent. Sous les yeux, deux rides à peine esquissées, dessinent des pattes d'oie en angles aigus, qui vont rejoindre la naissance de la barbe. La moustache qui suit le tracé de la lèvre, retombe aux commissures, en forme de crocs. Ia bouche est large, profondément creusée mais légèrement entr'ouverte. Une impression dramatique, de tension et de douleur mêlées, se dégage de ce premier masque. Jean-Pierre Cèbe $^{3}$, au moment de la découverte de ces sculptures, proposa d'y voir le personnage d'Amphitryon, interprétation que nous remettons en cause plus loin.

\section{MASQLE 2}

Fig. 44

Hauteur maximale conservée : $45 \mathrm{~cm}$

Largeur de la base : $54,5 \mathrm{~cm}$

Épaisseur : $18 \mathrm{~cm}$

Le deuxième masque est moins bien conservé puisque la partie supérieure de l'onkos a presque entièrement disparu. C'est un visage d'allure féminine, plus longiligne, portant une perruque semblable à celle du personnage précédent. Mais la coiffure était différente : un bandeau recouvrait la partie inférieure, descendant jusqu'au front et retombait en larges plis sur les côtés du visage. Ce bandeau est retenu par une guirlande de feuilles et de fleurs, dont il subsiste quatre éléments identifiables. Les indentations des trois feuilles conservées font penser à du lierre et la fleurette à cinq pétales circulaires est certainement du corymbe. Le nez est mutilé, les yeux sont allongés, cernés par une large paupière, sans signe de pupille. Les deux traces qui apparaissent sur le front sont dues à des accidents et les trois marques en creux qui apparaissent sur les joues sont

3 J.-P. Cèbe, in: L. Lambert (dir.), Le mausolée de Cucuron, Apt, 1982, p. 14. 

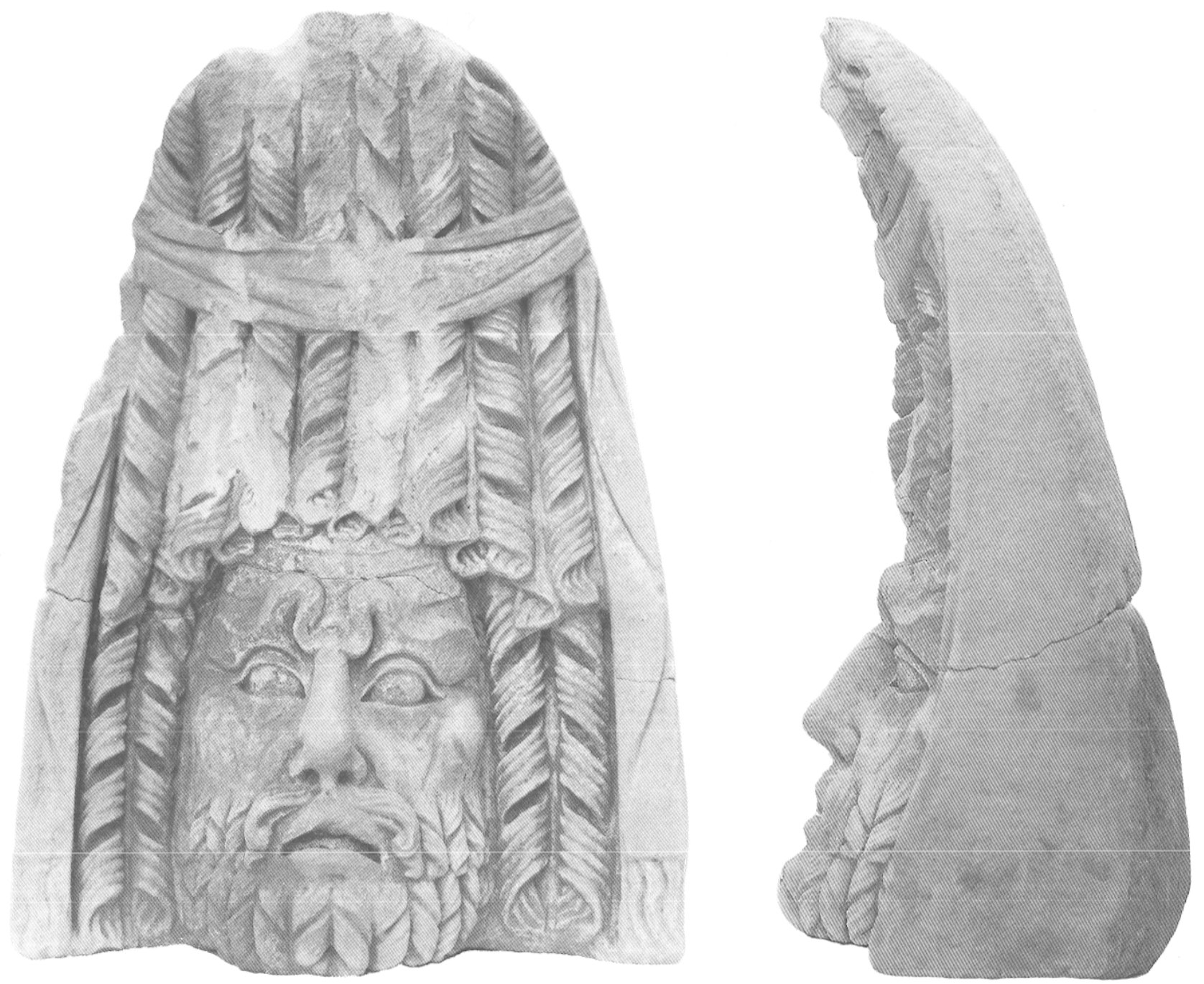

Fig. 43 - Masque 1 : vieillard tragique.

des défauts de la pierre plutôt que des larmes, comme on l'avait cru un moment. La bouche est fine, faiblement ourlée, formant une cavité profonde. Le menton est prononcé, un peu relevé en avant. J.-P. Cèbe y voyait Mégare, l'épouse d'Hercule.

\section{Masque 3}

Fig. 44

Hauteur maximale conservée : $40 \mathrm{~cm}$

Largeur de la base : $53,5 \mathrm{~cm}$

Épaisseur : $20 \mathrm{~cm}$

Plus dégradé que les précédents, le troisième masque a une coiffure très caractéristique. Il n'en reste que quelques boucles torsadées le long des joues, qui se réunissent en longues mèches striées sur les tempes et se rejoignent au milieu du front où l'on distingue le bas de la coiffe. Sur le côté gauche, deux serpents semblent sortir de la chevelure et dardent leurs têtes triangulaires vers le visage. L'œil et la gueule des reptiles sont finement incisées. A droite, seule la partie inférieure du premier serpent est conservée, les restes de la tête d'un second reptile étant à peine perceptibles à la hauteur de la tempe. Le visage est plein, lisse, le nez peu accentué, les yeux sans expression sous des orbites en faible saillie. L'hypothèse de J.-P. Cèbe était d'y voir le masque de Lyssa, la déesse grecque de la folie meurtrière.

\section{MASQUE 4}

Fig. 44

Hauteur maximale conservée : $70 \mathrm{~cm}$

Largeur : $60 \mathrm{~cm}$

Le quatrième masque est un Hercule, aisément identifiable, malgré les altérations de la pierre qui ont rongé une partie des traits du héros. L'onkos, recouvert de la dépouille du lion, est recourbé vers 


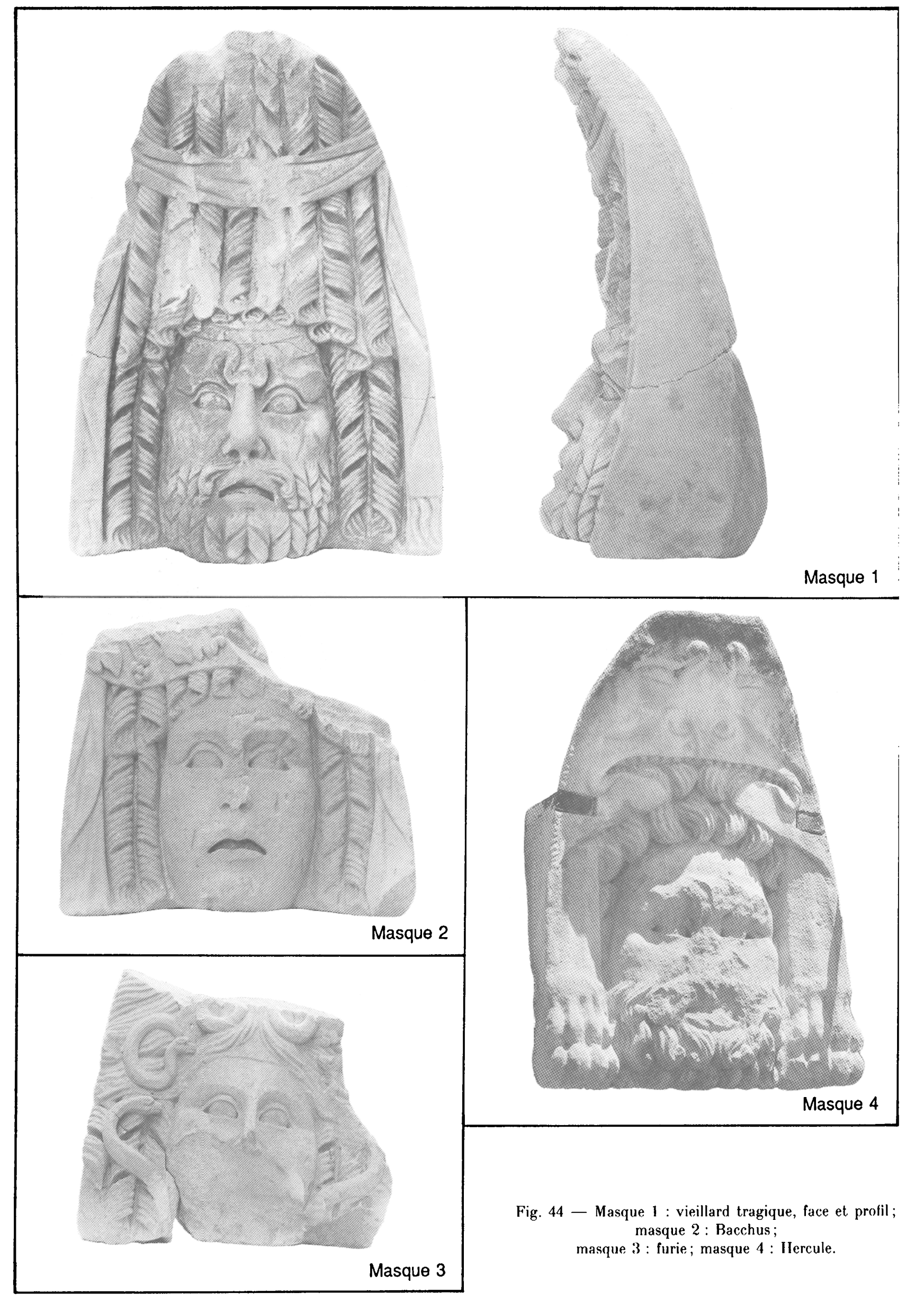


l'avant et permet de distinguer encore les cavités orbitales en haut du mufle de l'animal. De chaque côté du visage, les pattes aux puissantes griffes, retombent massivement. Sur le front, les cheveux forment deux séries de boucles superposées en coquilles. Le front est large et bien dégagé ; les yeux, les sourcils, le nez et la bouche sont difficiles à discerner, mais les arcades sourcilières semblent avoir été très saillantes et les points lacrymaux fortement marqués. La barbe est presque intacte, courte, formée de petites boucles épaisses, dont les extrémités recourbées s'opposent deux à deux.

I.'identification de ces quatre pièces comme Amphitryon, Mégare, Lyssa et Hercule, qu'avait proposée J.-P. Cèbe, ne va pas sans difficulté. Pour le masque d'Hercule, la dépouille léonine exclut toute mise en question, mais les trois autres sculptures ne sont pas pour autant les images des principaux protagonistes de l'Héraklès d'Euripide ou de sa "version" latine, l'Hercules furens de Sénèque. On peut noter d'abord qu'Hercule est l'un des héros les plus fréquemment représentés dans la plastique funéraire, en dehors de tout rattachement à une pièce de théâtre quelconque, grecque ou latine. Rappelons seulement, parmi tant d'exemples les masques de Servane, près de Glanum ${ }^{4}$, de Talmond en Vendée ${ }^{5}$ ou en Italie celui d'Ostie ${ }^{6}$, celui de Rome $^{7}$, ou ailleurs dans l'empire, les masques de I.indos ${ }^{8}$ ou celui du Musée d'Izmir ${ }^{9}$. Pour le masque féminin aux cheveux entremêlés de serpents, l'identification avec la Lyssa de la pièce d'Euripide est également recevable, puisque les vers $883-884$ l'assimilent nommément avec Gorgone "aux cent têtes sifflantes». Cependant, si l'on n'accepte pas d'emblée que les masques de Cucuron soient une illustration de la pièce grecque, on peut tout aussi bien admettre qu'il s'agit seulement d'une Furie, comme par exemple le masque trouvé à Dauphin (Alpes-deHaute-Provence) ${ }^{10}$. Nos réticences se précisent avec

4 Servane (Bouches-du-Rhỏne), Espérandieu, XII, 7888, 7889. Voir infra, note 48.

5 Cf. infra, note 49.

6 M. Bifaer, The Ilistory of the Greek and Roman

Thealer, Princeton, New-Jersey, 1961, p. 244, fig. 805.

7 Ibid., p. 102, fig. 393 et note 82, p. 289.

8 F. Hil.ter von Gärtringev, Heraklesmaske aus Lindos, Strena IIelbigiana, Leipzig, 1900, p. 137.

$9 \mathrm{Vu}$ par nous en 1975; semble inédit.

10 Gallia, 12, 1954, 2, p. 444; - Espérandieu, 15, 8628. Le lieu de trouvaille donné par les informations archéologiques de Gallia est erroné. La découverte a èté faite à la ferme de la "Camargue" (et non de la "Crau»), comme le précise la revue Alpes de Lumière, vol. 49, Le pays de Forcalquier, p. 69, s.v. Dauphin. Voir aussi R. LoIsy, Le mascaron de Dauphin, Bulletin *Les Amis du Vieux Riez *, 27, le masque de vieillard de tragédie. Aucun détail iconographique ne permet d'assurer que nous aurions affaire à Amphitryon, car l'Antiquité ne nous a livré aucune image du père d'Hercule susceptible de fournir des éléments de comparaison ${ }^{11}$. Ce serait donc ici la première et, actuellement, la seule image de ce héros. D'autre part, si l'on s'attache au seul trait caractéristique de ce masque, le froncement de sourcils particulièrement accentué, nous constatons qu'il ne lui appartient pas en propre. Michela Sassi Settis $^{12}$ l'a rappelé récemment à propos de l'étude de L. Bernabo Brea sur les masques de Lipari ${ }^{13}$, en citant un texte pseudo-aristotélicien ${ }^{14}$ relatif aux mouvements de la physionomie : c'est une des expressions fondamentales typiques de la douleur et de la colère dans les masques tragiques ${ }^{15}$. Par conséquent, plutôt qu'un très hypothétique Amphitryon, nous y voyons plus volontiers le Héros tragique, figuré par une image très générique.

Enfin, ce qui affaiblit considérablement la proposition de J.-P. Cèbe est son identification du masque féminin dans lequel il reconnait Mégare. Nous y voyons, pour notre part, un Bacchus. En effet, aux traits d'allure féminine du dieu, qui sont constants dans son image, s'ajoute sa couronne de feuilles de lierre et de corymbes, attribut spécifique de Bacchus. On le comparera, pour s'en convaincre, à deux Bacchus très proches, trouvés en Gaule, hors contexte, mais qui sont certainement des masques de mausolées comparables à celui de Cucuron : l'un est au Musée de Vienne (Isère) ${ }^{16}$ (fig. 45), le second, mis $\mathrm{au}$ jour près de Vaison-la-Romaine (fig. 46) ${ }^{17}$ est conservée au Musée Calvet d'Avignon. Tous deux comportent des feuilles analogues de lierres et de corymbes et ont été identifiés comme des Bacchus.

mars 1987 , p. $3-9$ et 28 , juin 1987 , p. 5-10. Le masque est conservé au Musée de Riez (Alpes-de-Haute-Provence).

11 Cf. l'article "Amphitryon", Lexicon iconographicum mythologiae classicae, II, p. 736 (A. Dale Trendall).

12 M. SAssi Setris, Quaderni di Storia, 19, 1984, p. 277-278.

13 L. Bernabo Brea, Menandro e il teatro greco nelle terracotte liparesi, Gênes, 1981.

14 Problemala, XXXI, 7, 15-20. Pour la valeur de l'expression donnée par les sourcils, cf. également le traité de Physiognomonie d'un anonyme latin, publié par J. André, Paris, Les Belles Lettres, 1981, p. 65, note 3.

$15 \hat{\omega} \sigma \pi \varepsilon \rho \tau \dot{\alpha} \tau \rho \alpha \gamma i x \dot{\alpha} \pi \rho \sigma \sigma \omega \pi \alpha$.

16 Espérandieu, I, 396: - E. Will, La sculpture romaine au musée lapidaire de Vienne, Vienne, 1952, p. 50, $\mathrm{n}^{\circ} 98 \mathrm{~A}$.

17 Espérandieu, I, 287; - J. Sautel, Vaison dans l'antiquité, Lyon, 1926, p. 221, no 513 et Vaison-la-Romaine, recueil documentaire illustré, Lyon, 1955, pl. 54,2; II. MöвıUs, Interpretatio celtica, Uber Masken an provinzialrömischen Grabmalern, Bonner Jahrbucher, 161, 1961, p. 142 


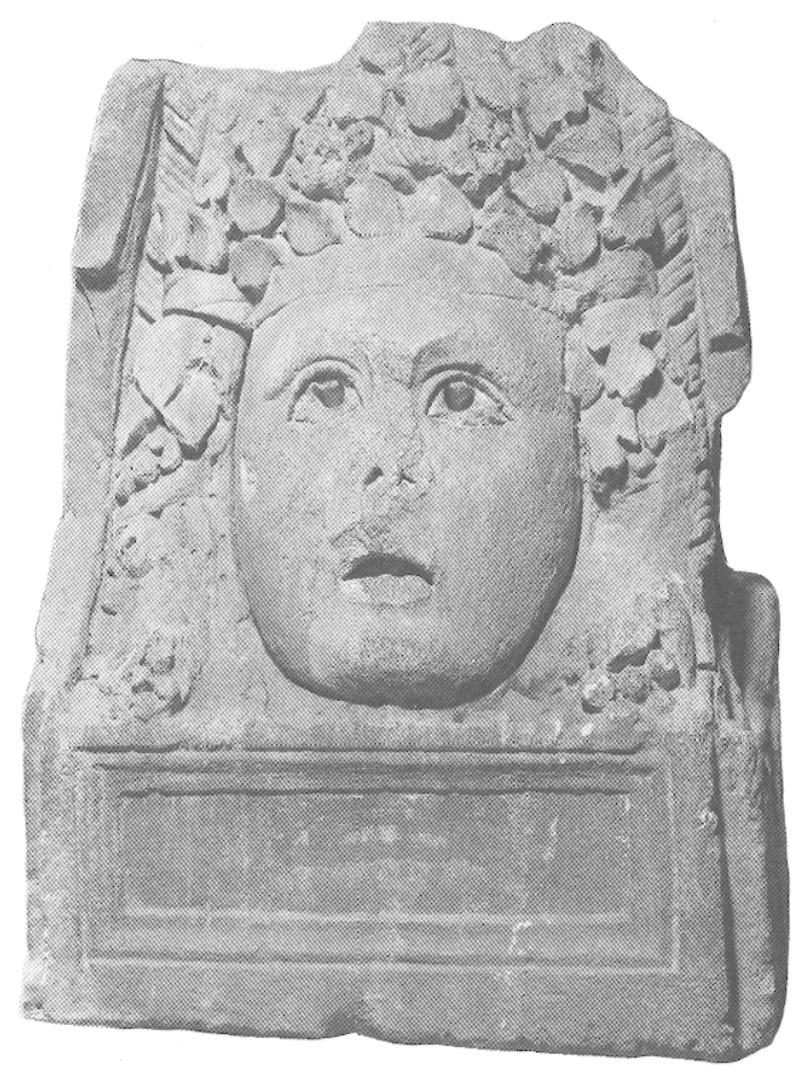

Fig. 45 - Vienne (Isère). Masque de Bacchus.

Mais il faut rapprocher surtout d'une série de quatre masques sculptés en faible relief sur un tombeau de la via Appia près de Rome ${ }^{18}$. On y reconnaît un Bacchus identique, avec la même coiffure ${ }^{19}$. Ce tombeau romain fournit d'ailleurs la comparaison la plus significative pour comprendre les masques de Cucuron. Les quatre figures présentées constituent un mélange assez hétérogène : Sol, Jupiter, Bacchus et Hercule, mais elles ont pour point commun d'avoir l'onkos des masques dramatiques, témoigna-

et pl. 28,3; - T. B. L. Webster, Monuments Illustrating Tragedy and Satyr Play, $2^{\circ}$ ed., University of London, Institute of classical studies, Bull. Suppl., no 20,1967, p. 133, no 418; H. LAVAGNE, in : Le goût du théátre à Rome el en Gaule romaine, catalogue d'exposition, Lattes, 1989, p. 213, no 99 et fig. 99, p. 217.

18 W. von Syoow, Eine Grabrotunde an der Via Appia antica, Jahrbuch des deutschen archäologischen Instituts, Berlin, XCII, 1977, p. 241-319, spec. p. 300-304, fig. 32-33.

19 On rapprochera également du masque funéraire encastré dans la façade de la cathédrale de Saint-Bertrand-deComminges (Revue du Comminges, 1966, p. 137-141). Ce type de masque a èté imité à l'époque médiévale (V. Lassalle, Une imitation d'un masque scénique antique sur un chapiteau roman de Brioude, Revue Archéologique du Centre, XIII, 51-52, 1974, p. 229-233).

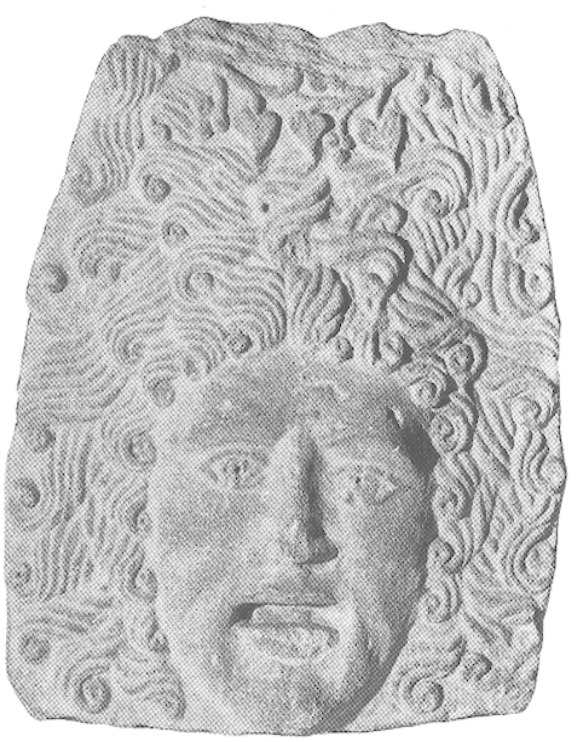

Fig. 46 - Rasteau (Vaucluse). Masque de Bacchus.

ge remarquable de l'influence du théâtre sur l'image divine elle-même, en dehors de tout rôle scénique particulier.

L'hypothèse de J.-P. Cèbe, qui exige au départ un postulat discutable ainsi qu'une identification impossible (le personnage-clé de Mégare) ne saurait donc être retenue. Au reste, si nous replaçons les figures de Cucuron dans la série des masques funéraires déjà connus, nous constatons que le lien avec le théâtre est fondé simplement sur le thème bien connu du mimus vitae dont on a souvent montré la valeur dans le décor funéraire ${ }^{20}$.

L'importance des masques dans l'art des tombeaux est bien connue ${ }^{21}$ et chacun sait qu'ils constituent un des éléments les plus répandus des stèles $^{22}$, des autels ${ }^{23}$, des urnes cinéraires ${ }^{24}$ et, à partir du $\mathrm{II}^{e} \mathrm{~s}$., des sarcophages où ils forment les

20 Cf. à propos des masques du mausolée des Julii à Glanum, P. Gros, Note sur deux reliefs des Antiques de Glanum : le problème de la romanisation, Revue Archéologique de Narbonnaise, XIV, 1981, p. 170-171. Pour ce thème, d'un point de vue plus général, voir A. Allrogren-Brdre., Maskendarstellungen in der Römisch-Kampanischen Wandmalerei, Münich, 1974, p. 70-73 et M. Gigante, Civilla delle forme lellerarie nell'antica Pompei, Naples, 1979, p. 128-132.

21 Nous renvoyons ici à notre étude parue dans le catalogue Le goût du théâtre ... cité supra, note 17, p. 209-218.

22. Exemples des stèles de la nécropole de Trion à Lyon, pour s'en tenir à une série connue (Espérandieu, III, 17821787).

23 W. Altmann, Die Grabaltäre der Kaiserzeit, Berlin, 1905 , p. $150, \mathrm{n}^{\circ} 177$, fig. 121 , et p. $185, \mathrm{n}^{\circ} 267$, fig. 155 .

24 Espérandieu, I, 281, III, 1853 et 1789 . - Dans Altmann, op. cit., p. 110-111, n"105, fig. 90-91. 
figures d'angles des couvercles ${ }^{25}$. Beaucoup nous sont parvenus sans contexte sûr, et notamment pour les exemplaires en terre cuite, il est difficile de faire le départ entre les acrotères de petits monuments ${ }^{26}$ et les ex-voto renfermés dans les tombes comme à Lipari ${ }^{27}$. Nous ne nous attacherons qu'aux masques en pierre, dits de type "colossal», dont une des caractéristiques est leur grande taille (en moyenne $70 \mathrm{~cm}$ de haut, pouvant aller jusqu'à $90 \mathrm{~cm}$ ), l'autre étant la courbure de leur face arrière, laquelle se prolonge par un large empattement destiné à leur donner une base stable sur les monuments où ils sont placés, sans aucune trace de crampons de scellement. Ils se trouvaient, en effet, soit aux angles de l'enclos funéraire comme dans la tombe dite des Platorini ${ }^{28}$ ou dans un des mausolées de Trion (Lyon) ${ }^{29}$, soit sur le monument lui-même, généralement aux quatre coins du toit en pyramide, comme on le voit par exemple, sur les mausolées de Neumagen conservés au Musée de Trèves ${ }^{30}$ et sur les restitutions du mausolée des Solonii à Lyon ${ }^{31}$ ou sur celui de Faverolles (Haute-Marne) ${ }^{32}$. Dans le cas de Cucuron,

25 T. Brennecke, Kopf und Maske, Untersuchungen zu den Akroteren an Sarkophagdeckeln, Berlin, 1970. Également, R. Turcan, Masques corniers d'orientaux : Attis, Ganymède ou Arimaspe, dans Mélanges de philosophie, de littérature et d'histoire ancienne offerts $\grave{a} P$. Boyancé, Collection de l'École française de Rome, 22, 1974, p. 721-747. D'autres exemples dans Espérandieu, I, 502, 544, 553; XI, 7697, etc. Quelquesuns d'entre eux avaient été réunis lors de l'exposition Le masque de théâtre dans l'antiquité classique, catalogue édité par P. Ghiron-Bistagne, Arles, 1986.

26 Parmi de très nombreux exemples, citons quelques cas relevés dans Gallia: 11,1953, p. 149, fig. $3 ;-12,1954$, p. 231 , fig. $20 ;-23,1965$, p. 376 , fig. 43 ; p. 402 , fig. 29 ; 30,1972 , p. 104 , fig. $18 ;-33,1975$, p. 435 , fig. $32 ;$ p. 440 , fig. 1 ; p. 478 , fig. 23.

27 Cf. supra. note 13.

28 F. Silvfistrini, Sepulcrum M. Artori Gemini, la tomba detla dei Platorini nel Museo nazionale romano, Rome, 1987, p. 30-37.

29 A. Allomer, P. Dissard, Trion, II, Antiquités découverles en 1885-86 au quartier de Lyon dit de Trion, Lyon, 1888, p. 303-304. Quatre masques, comme à Cucuron furent trouvés «à l'angle nord-est du mur de clôture de l'enceinte funéraire".

30 R. Schindier, Führer durch das Landesmuseum Trier, Trèves, 1986, p. 106-108, fig. 329-342.

31 II. Käнı,FR, Die rheinischen Pfeilergrabmäler, Bonner.Jahrbücher, 139, 1934, p. 159, 171 et fig. 3.

32 Gallia, 41, 1983, p. 389, fig. 31 . Le projet de restitution, en; cours d'ètude, nous a été aimablement communiqué par l'un des fouilleurs du monument, M. S. Février. Voir, de ce mème auteur, Le mausolée gallo-romain de Faverolles, fouilles de 1983 et 1984, Bulletin de la Société des Sciences Nalurelles et d'Archéologie de la Haute-Marne, XXII, 10, 1985, p. 176-196 et ibid., XXIII, 18, 1987, p. 349-366, notamment fig. 15 . il est impossible de se prononcer entre ces deux emplacements possibles puisque nous ignorons l'aspect exact du monument lui-même. Étant donné leur grande taille et la courbure accentuée des sculptures, nous pensons que, pour des raisons optiques, ils devaient, en tout cas, être placés à une certaine hauteur.

Un bref rappel des principaux exemples permet de mieux saisir la place de ceux de Cucuron ${ }^{33}$. La série la plus importante est celle du Musée de Vienne $^{34}$, même si les quatre masques réunis au Musée Saint-Pierre ne proviennent peut-être pas du même monument tant leurs différences typologiques et stylistiques paraissent importantes ${ }^{35}$. A Vaison-laRomaine, huit masques ${ }^{36}$ ont été mis au jour depuis le $\mathrm{xIX}^{\mathrm{e}} \mathrm{s}$. mais, comme à Vienne, leur provenance précise est inconnue. Trois autres sont conservés à

33 Nous laissons de côté les masques qui appartiennent uniquement au décor architectural des théâtres, dont la plus belle série a été récemment étudiée par P. Ciancio Rossetro, Le maschere del teatro di Marcello, Bulletino della Commissione Archeologica Communale di Roma, 87, 1980-81 (1982), p. 20-30. Il faut inclure dans cette série le masque de théâtre (Espérandieu, III, 2498) conservé aujourd'hui au Musée Calvet à Avignon. Il faisait partie de la collection de l'érudit aixois $\mathrm{F}$. Sallier, dont on sait qu'elle fut constituée à partir d'achats faits en Italie. Le style italique de cette pièce incline à en faire un objet venu de Rome.

34 Le Musée Saint-Pierre de Vienne (Isère) renferme, outre l'exemple cité supra, note 16: - un masque de vieillard barbu (Espérandieu, I, 398; - E. Will, op. cit., p. 50, no $98 \mathrm{D} ;-$ Möвius, op. cit., p. 149 et pl. 29,3). • un masque féminin à longues boucles tuyautées (Espérandiev, I, $397 \mathrm{~A} ;-$ E. WrLL, p. 50, no 98B). • un masque féminin à deux rangs de bouclettes sur le front (Espérandieu, I, 397B; -E. WiLL, p. 50, no 98C).

35 Deux d'entre eux ont un socle mouluré, qui ètait peut-être destiné à porter une inscription.

36 Outre l'exemple cité supra, note 17, on compte sept masques à Vaison-la-Romaine ou qui ont été trouvés dans les environs immédiats : - Espérandeu, I, 288,2;-SaUtel, op. cit., p. 221 et pl. 28,1 ; - WeBster, op. cit., p. 133, no 416 ; - H. Lavagne, op. cit., p. 213 , no 100 et fig. 100 , p. 217. - Espérandieu, I, $292 ;$ - SaUtel, p. 221, no 515 ; - Möbius, p. 142 et pl. 28, 4 ; - Y. De Kisch, Fr. Salviat, Vaison-la-Romaine, Coll. Guides archéologiques de la France, Paris, 1984, p. 63, pl. couleurs; - H. Lavagne, op. cit., p. $213, n^{\circ} 98$ et fig. 98, p. 216 . - Espérandieu, I, 288, 1 ; Sautel, p. 220 , no $510 ;$ - Möbius, p. 142 et pl. 28,2 ; Webster, p. 133, no 417 ; - H. LaVAgne, op. cit., p. 214, $\mathrm{n}^{\circ} 101$ et fig. 101, p. 217. - SaUtel, p. 220-221, no 511 . . Sautel, p. 220-221, no 514. - Sautel, p. 220-221, $\mathrm{n}^{\circ}$ 516. - Masque trouvé dans la maison Ramon, cf. Gallia, 42,1984, p. 430.

Nous écartons de ce relevé le masque Espérandieu, I, 307 , qui est un simple acrotère et $\mathrm{X}, 307$, qui appartenait au décor architectonique du théâtre. On ne comptera pas non plus la plaque rectangulaire Espérandieu, IX, 6770 qui est du type des oscilla. 
Aix-en-Provence ${ }^{37}$, un à Servane ${ }^{38}$, un à Glanum ${ }^{39}$, un à Dauphin ${ }^{40}$, deux à Fréjus ${ }^{41}$, deux à Gigondas ${ }^{42}$, deux à Arles $^{43}$ et cinq à Narbonne ${ }^{44}$. Le seul ensemble dont l'origine exacte est attestée est celui de Sisteron (Alpes-de-Haute-Provence) dont les trois exemplaires ${ }^{45}$ furent dégagés dans les fouilles de sauvetage d'un mausolée à l'entrée de la ville en 1949. Nous ajouterons à cette série provençale un masque colossal, conservé aujourd'hui au Musée de la Civilisation gallo-romaine à Lyon ${ }^{46}$ et qu'on croyait avoir été trouvé dans les fouilles de la nécropole de Trion en 1885-1886. Nous avons montré qu'il provenait, en fait, d'un monument funéraire d'Uchaux (Vaucluse) ${ }^{47}$.

37 Espérandieu, I, 109 repris dans Espérandieu, Lantier, XII, 7861; - Möbius, p. 142 et pl. 29,1 ; Webster, p. 134, no 419 ; - R. Ambard, Aix romaine, Aixen-Provence, 1984, p. 35, fig. 16 ; - H. Lavagne, p. 213, no 97 et fig. 97 , p. 216. - Espérandieu, XV, 8646; Gallia, 12, 1954, p. 298, fig. 11 ; - Möвıus, p. 142 et pl. 27, 2; - Webster, p. 134 , no 421 ; - R. Ambard, op. cil., p. 38 , fig. 17. - Un fragment de masque est conservé dans les réserves du Musée Granet à Aix-en-Provence et paraît inédit.

38 Cf. supra, note 4.

39 Espérandieu, XII, 7875 , pl. XIII. Nous ne retenons pas le masque qui sert de déversoir à la piscine froide des thermes de Glanum (Espérandiru, XII, 7861, pl. XI et II. Rolland, Fouilles de Glanum, Saint-Rémy-de-Provence, $1^{\text {er }}$ suppl. à Gallia, Paris, De Boccard, 1946, p. 51-53, fig. 4142) car rien ne fait supposer qu'il puisse s'agir d'un réemploi de masque funéraire. Ces cas de réemplois sont très rares : un exemple dans un balneum d'Ampurias (G. Fabre, I. Roda, M. Mayer, Inscripciones alienae en museos y colleciones, Ampurias, 44, 1982, p. 231, fig. 43 et un autre dans une fontaine de Pompéi (H. Eschebach, Katalog der Pompejanischen Laufbrunnen Antike Welt, 3, p. 21-26).

40 Cf. supra, note 10.

41 Un exemplaire provient des fouilles du théâtre (A. Courssin et alii, Forma Orbis Romani, Carte archéologique de la Gaule romaine, II, Var, Paris, 1932, no 17), un autre a été trouvé dans les fouilles du Clos de la Tour, avant 1959 et est conservé au Musée de Fréjus (inédit?).

42 Deux masques féminins, inédits. Ils proviennent de la collection Raspail et étaient primitivement. conservés au Château Raspail près de Gigondas (Vaucluse). Ils décorent aujourd'hui une propriété privée de la région. Leur authenticité nous parait discutable.

43 Espírandieu, IX, 6727; - Mörius, p. 142 et pl. 29,$2 ;-$ WeBster, p. 134, no 420 . Le second exemple est Espérandifu, I, 224 et Chr. Landes, in : Le goût du théâtre ..., op. cit., p. 187, no 76 et fig. 76, p. 191.

44 Espérandieu, I, 754 et 755-758.

45 Espérandieu, XV, 8625-8627; - Gallia, 7, 1949, p. 83 et fig. $6-8$, p. 85 . Dans Mörius, p. 149 , pl. $35,1-2$.

46 Espérandieu, très curieusement, ne paraît pas l'avoir connu. Dans Möвius, p. 150 , pl. 34,1 ; - Werster, p. $134, \mathrm{n}^{\circ} 428$.

47 H. Lavagne, op. cit., p. 214, no 102 et fig. 102, p. 217.
Par rapport à la Narbonnaise, les autres régions de la Gaule sont plus pauvres ou offrent des pièces dont l'authenticité est parfois douteuse. En effet, dès le $\mathrm{XvII}^{\mathrm{e}} \mathrm{s}$., la mode de ces grandes figures de pierre, servant de départs d'escaliers ou de mascarons de fontaines, s'est répandue dans l'architecture des jardins et les réemplois antiques voisinent avec les œuvres modernes ${ }^{48}$. Pour l'Aquitaine, on citera le masque d'Hercule du château de Talmond ${ }^{49}$, le masque trouvé en réemploi à Saintes ${ }^{50}$, les deux cas de Castelnau-Montratier ${ }^{51}$ et de LabastideLévêque $^{52}$; en Lyonnaise, les figures colossales de Chorey ${ }^{53}$ ont suscité bien des doutes ${ }^{54}$ et font penser à un exemplaire analogue trouvé à Montceau-lesMines $^{55}$, représentant, une fois encore, Hercule. Plus sûrs, quoique notablement restaurés, sont les masques de la nécropole de Lyon ${ }^{56}$ ainsi que la grande

48 Comme le fait remarquer F. Braemer (Bronzes romains, problème de géographie, dans Actes du Colloque Toreutik und figürliche Bronzen römischer Zeit, Berlin, 1980, 1984, p. 85, n. 179). Pour lui l'Hercule de Servane (cf. supra, note 4) et les têtes de Chorey (cf. infra, note 53) ne "sont pas antérieures au Xvi ${ }^{\mathrm{e}}$ siècle".

49 Dans Gallia, 15, 1957, p. 220, fig. 16. Conservé aujourd'hui à l'Écomusée de la Vendée, au Puy-du-Fou. Selon F. Ribemont, il s'agirait, en fait, d'une pierre aux quatre dieux; nous n'avons pu voir ce masque.

50 Espérandieu, II, 1357-1358; - Mörius, p. 142 et pl. 33,3. Egalement L. Maurin, Sainles antique, Saintes, 1978, p. 149 et fig. $35,1,2$.

51 Ils se trouvent aujourd'hui au château de la Vernède (commune de Sauveterre, Tarn-et-Garonne). Cf. Gallia, 22, 1964 , p. $460-461$ et fig. 44.

52 Espérandieu, XIII, 8188 et pl. XXXVII.

53 Espírandieu, Lantire, XV, 9051 et Gallia, 5, 1947, p. 427-433. Dans Möвıus, p. 152-153 et pl. 35,3 et 4. $\mathrm{S}$. Deyts les a inclus dans son catalogue des sculptures du Musée de Dijon (Musée archéologique, sculptures gallo-romaines, mythologiques et religieuses, Paris, 1976, $\left.\mathrm{n}^{\circ \times} 286-288\right)$. On leur comparera le masque de Chagnon (Espŕrandieu, II, 1343 ; Mörius, p. 152).

54 Cf. F. Braemer, op. cil., p. 85.

55 Gallia, 9, 1951, p. 61, fig. 2.

56 Cf. supra, note 29. Il est difficile de faire le décompte exact et d'identifier les masques de Lyon, tant les informations qui les concernent sont imprécises. Dans le volume consacré aux fouilles de la nécropole de Trion, Allmer et Dissard (cf. supra, note 29) affirment que l'enclos funéraire ètait orné de quatre masques, en partie mutilés. Espérandieu (III, 1796) reprend cette affirmation et ne reproduit qu'une seule figure (masques tragiques repris dans Möвlus, p. 150 et pl. 33, 4 et Werster, p. 134, no 427). Mais tous les auteurs ne donnent que deux images de masques, dont celle d'un Cyclope, qui ne provient certainement pas de la fouille de Trion en 1885-1886. En effet, une enquête de Gérard Bruyère (recherches inédites) a montré que ce masque avait été trouvé entre 1808 et 1809 au quartier des Massuts (voir les manuscrits inédits de F. N. Cochard, conservés à la Bibliothèque municipale de Lyon, ms. 2382, s.v. "musée lapidaire de Lyon", p. 26). - 
figure de Polyphème trouvée au début du premier Empire. En Gaule Belgique, on relève un cas à Naix $^{57}$, deux exemplaires à Reims ${ }^{58}$ et le groupe, en cours de publication, de Faverolles ${ }^{59}$. Pour étendre l'enquête au-delà des frontières de la Gaule, citons quelques exemples, en Germanie Supérieure, à Strasbourg $^{60}$, au Musée de Cologne ${ }^{61}$, à Darmstadt ${ }^{62}$, à Bonn $^{63}$, au musée de Bruxelles ${ }^{64}$, à Zurich ${ }^{65}$, sans oublier la série déjà citée de Neumagen au Musée de Trèves ${ }^{66}$.

De ces comparaisons, plusieurs conclusions se dégagent. En premier lieu, on notera la répartition géographique des trouvailles qui est déjà révélatrice : la Narbonnaise vient largement en tête avec 37 exemples, puis la Gaule Belgique avec 7 exemples, la Lyonnaise avec 5 exemples et l'Aquitaine avec 5 cas. On peut dire que la province de Narbonnaise, et plus spécialement encore, la Provence intérieure jusqu'à Vienne, est, de loin, la région la plus riche en monuments de ce type. La proximité de l'Italie en est certainement la cause essentielle, même si les masques en Italie sont moins nombreux qu'on l'attendrait ${ }^{67}$. L'hypothèse de Hans Möbius ${ }^{68}$

Marcel Le Glay (Notes d'épigraphie et d'archéologie lyonnaises, Travaux édités sous les auspices de la Ville de Lyon, V, 1976, p. 9 et fig. 3) parle de "deux masques cyclopiques", sans préciser quel serait le second. Le nombre des masques lyonnais paraît, sous réserve d'inventaire dans les collections du Musée, ètre de 5 .

57 Espérandieu, VI, 4661 ; - Möbius, p. 142 et pl. 27,4 .

58 Inédits.

59 Cf. supra, note 32. Six masques, en partie conservés.

60 Espírandiev, $\mathrm{X}, 7310$.

61 Espŕrandieu, ViII, 6652, 6554; - Möbius, p. 143

et pl. 28,4 . - Le n" 5016 (Trèves) parait ètre un faux.

62 Möвıиs, p. 143 et pl. 27,3.

63 Espérandieu, VIII, 6260; - Möbius, p. 142.

64 Bloc d'Herstal, près de Liège, conservé au Musée du Cinquantenaire à Bruxelles (Esprirandieu, V, 4011 ; Möвıus, p. 143).

65 Espŕrandifu, VII, 5453; - Mörius, p. 143.

66 Cf. supra, note 30.

67 Comme masque de type colossal provenant sûrement d'un tombeau, nous n'avons relevé que l'exemple du mausolée des Calpurnii près de la Porta Salaria à Rome (Birber, op, cit., p. 102, fig. 393 et p. 289, note 82). - Le Musée national des Thermes à Rome conserve un certain nombre de masques, provenant en particulier de la Villa d'Iladrien, mais leur localisation originelle est inconnue. Beaucoup devaient orner les différents théâtres de la Villa (Museo nazionale delle Terme, le sculture, I, 1, nos 7,8 et I, $2, n^{* *} 27,57,59$, etc.) On y ajoutera les deux masques de Pan du Musée archéologique de Venise qui nous paraissent avoir pu appartenir à un tombeau plutôt qu'à un sarcophage (R. Polacco, G. Traversari, Sculture romane e avori tardo-antichi e medievali del museo archeologico di Venezia, Collezioni e musei archeologici del selon laquelle la coutume celto-ligure des "têtes coupées" aurait pu créer un terrain favorable à ce goût pour les masques est à prendre en considération avec prudence, car il semble bien qu'à l'époque romaine, ce soit la "dimension théâtrale" qui donne sa signification essentielle à cette tradition décorative, plus qu'à une hypothétique "résurgence celtique».

Sur le plan stylistique, la datation des masques de Cucuron est délicate. Un point s'impose : par rapport à tous les cas que nous venons d'énumérer, ce sont incontestablement ceux dont la qualité plastique est la meilleure. Si l'on considère le traitement des boucles de l'onkos, la différence avec les exemplaires les mieux venus comme ceux de Vienne, est éclatante. Les rouleaux sont très finement travaillés à Cucuron avec des creux qui les détachent, alors qu'à Vienne, ils sont à peine séparés du plan de taille et rendus de manière raide et uniforme. Dans tous les autres cas, ces "anglaises" sont traitées de manière très stéréotypées, "en bâtons de sucre d'orge", pour reprendre l'expression des archéologues anglais ${ }^{69}$. Il en va de même pour le masque de la Furie, dont les serpents sont remarquablement animés, alors que dans la pièce comparable de Dauphin ${ }^{70}$ les reptiles sont presque indistincts dans la masse de la chevelure. Enfin, un trait particulier des sculptures de Cucuron les met à part dans la série : les yeux sont vides et saillants sous des paupières supérieures largement ourlées, ce qui s'oppose au traitement habituel des autres masques, dont les globes oculaires sont toujours creusés, tantôt largement, tantôt en lunules, l'iris étant souvent cerné d'un cercle. Cette manière de rendre l'œil plein sans marquer l'iris au trépan (dont l'usage n'apparaît d'ailleurs pas dans les masques de Cucuron) nous fait placer ces sculptures dans le dernier quart du $\mathrm{I}^{\mathrm{er}} \mathrm{s}$. de notre ère. Il est exact que ce trait ne constitue pas un indice chronologique absolument déterminant, mais au moins peut-on remarquer avec Fr. Braemer ${ }^{71}$ que le traitement de

Veneto, 31, Rome. 1988, nos 9-10, p. 33-36). En revanche, le masque trouvé Via Labicana près de Rome, que $M$. Bieber inclut dans sa liste (R.E., col. 2110, s.v. "maske») est, en fait, un simple motif de claveau (cf. Not. Scav., 1926, p. 294).

68 Möвıus, op. cit., p. 153-154.

69 Webster, op. cil., p. 134.

70 Cf. supra, note 10.

71 F. Braemer, Un apport de l'Égypte à la Narbonnaise dans la technique sculpturale, in: Alessandria e il mondo ellenistico-romano, Studi in onore di Achille Adriani, Rome, 1984 , p. $421-430$. 
l'iris creux, absent de Cucuron, l'est également des sculptures de l'Italie du Nord, notamment dans la région alpine. En particulier, il ne figure pas sur l'arc de Suze. Par conséquent, nous serions porté à voir dans les masques de Cucuron la main d'un artiste, sinon d'origine ilalique, du moins formé à la manière de l'Italie du début de l'Empire, alors que dans le cas des exemples de Vienne ou de Vaison-la-Romaine, on a l'impression d'un style plus "gallo-romain" et sans doute déjà du $\mathrm{II}^{\mathrm{e}} \mathrm{s}$.

Henri Lavagne.

\section{LES INSCRIPTIONS}

\section{INSCRIPTIONS DÉCOUVERTES AU VOISINAGE DU MAUSOLÉE}

1 Trouvée en avril ou mai 1970. Conservée au musée de Cucuron.

Fig. 47. Plaque de calcaire rectangulaire brisée obliquement dans sa partie inférieure. Hauteur: $35,5 \mathrm{~cm}$; largeur : $50 \mathrm{~cm}$; épaisseur (incomplète) : $15,5 \mathrm{~cm}$. Hauteur des lettres : lignes 1 et $2: 5,5 \mathrm{~cm}$; ligne $3: 4,4$ à $5,2 \mathrm{~cm}$; ligne $4:$ incomplète.

Inédite. Révisée en novembre 1986.

$$
\begin{aligned}
& \text { [. ?] VCVTTECTO } \\
& \text { [. ?] COMMIVS } \\
& \text { [..]TTVRNINVS } \\
& \text { [-......- }] \mathrm{M}
\end{aligned}
$$

Au début de la 1. 1, la surface, endommagée, pouvait contenir une lettre avant le premier $\mathrm{V}$. Il est même probable qu'un caractère y était inscrit : en effet, à la 1.3 , il y avait certainement deux lettres avant le $\mathrm{T}$ initial partiellement conservé. La seconde de ces lettres se trouvait nécessairement à l'aplomb du premier $V$ de la 1 . 1. Or, la mise en page, contrastant avec la qualité relativement médiocre de la gravure, paraît avoir été très soignée, car la dernière lettre des 1.2 et 3 est gravée juste à la verticale du dernier signe de la l. 1. Il est donc présumable que la première lettre de la l. 1 était aussi exactement à l'aplomb de la première de la l. 3 . Il est possible que subsiste avant le $\mathrm{V}$ une très faible trace d'un fragment de haste, mais on ne peut l'affirmer, compte tenu de l'état de la pierre en cet endroit. La lecture [L]ucuttecto est cependant probable, car on ne peut guère envisager un autre premier élément de ce nom que lucu- $(=$ lugu- $)$, cf. infra;

1. 2: avant le gentilice Commius, il y a place pour un prénom abrégé en une lettre. Bien que le nom du dédicant ait pu ne comportcr qu'un gentilice

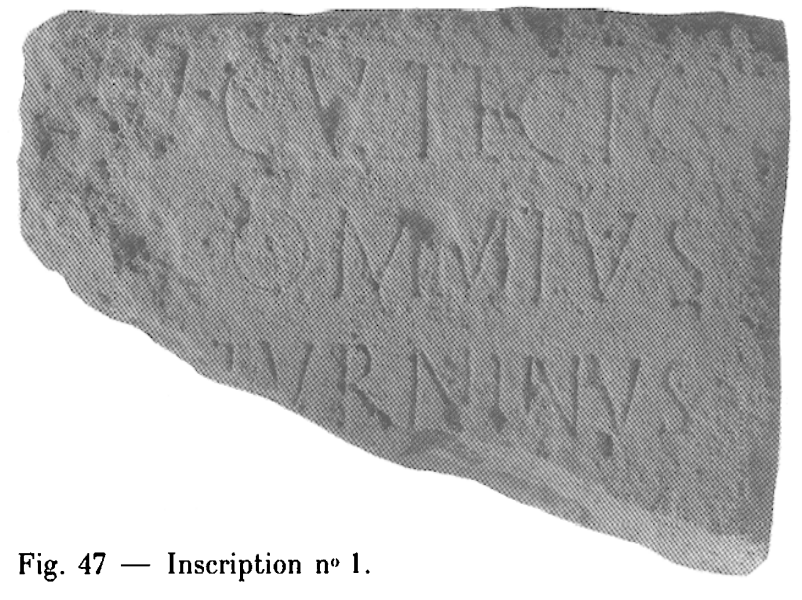

et un surnom, on supposera que le prénom était effectivement gravé pour la même raison de mise en page qu'on vient d'invoquer: l'initiale du prénom devait se trouver juste au-dessus de la première lettre de la 1. 3 ;

l. 3 : la restitution [Sa/turninus est évidente;

1. 4 : on distingue à la fin un fragment de barre oblique formant un angle aigu avec le haut d'une haste verticale. Il ne peut s'agir que de la fin d'un $M$.

On peut, à partir de là, restituer la formule votive bien connue: [u(otum) s(oluit) l(ibens] $m$ (erito). Au total, on comprendra ainsi l'ensemble du texte :

[L]ucuttecto / [.] Commius / [Sa]turninus / [u(otum) s(oluit) l(ibens)] m(erito).

"Envers Lucuttectus, [.] Commius Saturninus s'est acquitté de son vœu, de bon gré et à juste titre».

Lucuttectus : nom de divinité gallo-romaine jusqu'à présent inconnu. M. M. Lejeune, que nous 